TRANSACTIONS OF THE

AMERICAN MATHEMATICAL SOCIETY

Volume 351, Number 4, April 1999, Pages 1423-1443

S 0002-9947(99)02252-7

\title{
UNIVERSAL CONSTRAINTS ON THE RANGE OF EIGENMAPS AND SPHERICAL MINIMAL IMMERSIONS
}

\author{
GABOR TOTH
}

\begin{abstract}
The purpose of this paper is to give lower estimates on the range dimension of spherical minimal immersions in various settings. The estimates are obtained by showing that infinitesimal isometric deformations (with respect to a compact Lie group acting transitively on the domain) of spherical minimal immersions give rise to a contraction on the moduli space of the immersions and a suitable power of the contraction brings all boundary points into the interior of the moduli space.
\end{abstract}

\section{Preliminaries and Statement of Results}

An isometric immersion $f: S_{k}^{m} \rightarrow S_{V}, m \geq 2$, of the Euclidean $m$-sphere $S_{k}^{m}$ of constant curvature $k$ into the unit sphere $S_{V}$ of a Euclidean vector space $V$ is said to be a spherical minimal immersion if $f$ is minimal. By a result of Takahashi [11], $f$ exists iff $k=m / \lambda_{p}$ for some $p \geq 1$, where $\lambda_{p}=p(p+m-1)$ is the $p$-th eigenvalue of the spherical Laplacian $\triangle^{S^{m}}$ on $S^{m}:=S_{1}^{m}$, and, in this case, each component $\phi \circ f, \phi \in V^{*}$, of $f$ is a spherical harmonic of order $p$ on $S^{m}$; an eigenfunction of $\triangle^{S^{m}}$ with eigenvalue $\lambda_{p}$. We scale the metric on the domain to curvature one and call $f: S^{m} \rightarrow S_{V}$ a spherical minimal immersion of (algebraic) degree $p$. Because of the scaling, $f$ is a homothetic (minimal) immersion with homothety $\lambda_{p} / m$ :

$$
\left\langle f_{*}(X), f_{*}(Y)\right\rangle=\lambda_{p} / m\langle X, Y\rangle,
$$

where $X, Y$ are vector fields on $S^{m}$. Let $\mathcal{H}_{m}^{p}$ denote the Euclidean vector space of spherical harmonics of order $p$ on $S^{m}$ endowed with the $L^{2}$-scalar product (suitably scaled). The universal example of a spherical minimal immersion is the standard minimal immersion $f_{m, p}: S^{m} \rightarrow S_{\mathcal{H}_{m}^{p}}$ whose components (relative to an orthonormal basis in $\mathcal{H}_{m}^{p}$ ) are orthonormal. For $p=2, f_{m, 2}: S^{m} \rightarrow S_{\mathcal{H}_{m}^{2}}$ is the classical Veronese map. Unless relevant, we suppress $m$ from the notation and write the standard minimal immersion as $f_{p}: S^{m} \rightarrow S_{\mathcal{H}^{p}}$.

A fundamental problem raised by M.DoCarmo and N.Wallach [3] is to find lower bounds for the range dimension of spherical minimal immersions. The main result of this paper, Theorem 4, solves this problem by giving lower bounds in terms of the dimension of the domain, the degree, and differential geometric properties of the immersions such as symmetries and higher order isotropy.

The only previously known general lower bound was given by J.D.Moore [10] who proved that, for a spherical minimal immersion $f: S^{m} \rightarrow S_{V}$ of degree $\geq 2$, we have $\operatorname{dim} V \geq 2 m$. DoCarmo and Wallach conjectured [3] that the lower bound

Received by the editors April 20, 1997.

1991 Mathematics Subject Classification. Primary 53C42.

(C)1999 American Mathematical Society 
can be raised to $m(m+3) / 2$ (corresponding to the Veronese map) until N.Ejiri [5] showed the existence of a spherical minimal immersion $f: S^{3} \rightarrow S^{6}$ of degree 6. Subsequently, K.Mashimo [9] gave an explicit example of an $S U(2)$-equivariant spherical minimal immersion $f: S^{3} \rightarrow S^{6}$ of degree 6. Extending this example, a groundbreaking work of DeTurck and Ziller [2] resulted in an extensive list of new spherical minimal immersions (that gave rise to minimal imbeddings of all homogeneous space forms into spheres). These spherical minimal immersions are obtained by the so-called 'equivariant construction'; thus, they possess large groups of symmetries acting transitively on the domain. At the other extreme, Ch.Escher $[6,7]$ found a necessary and sufficient condition for the existence of imbeddings of 3-dimensional (inhomogeneous) spherical space forms (covered by lens spaces) into spheres by spherical minimal immersions. In particular, she showed that the inhomogeneous lens space $L(8,3)$ can be imbedded into $S^{190}$ by a spherical minimal immersion of degree 32.

Let $\mathcal{M}_{m}^{p}$ denote the moduli space of congruence classes of full spherical minimal immersions $f: S^{m} \rightarrow S_{V}$ of degree $p$. Here, fullness of $f$ means that the image of $f$ is not contained in a great hypersphere of $S_{V}$, and two spherical minimal immersions $f_{1}: S^{m} \rightarrow S_{V_{1}}$ and $f_{2}: S^{m} \rightarrow S_{V_{2}}$ are said to be congruent if there is an isometry $U: V_{1} \rightarrow V_{2}$ such that $U \circ f_{1}=f_{2}$. Given a spherical minimal immersion $f: S^{m} \rightarrow S_{V}$ of degree $p$, the space of components $V_{f}=\left\{\phi \circ f \mid \phi \in V^{*}\right\}$ is a linear subspace of $\mathcal{H}_{m}^{p}$. If $f$ is full, precomposition by $f$ gives an isomorphism $V^{*} \cong V_{f}$. The points in the interior of $\mathcal{M}_{m}^{p}$ correspond to spherical minimal immersions $f: S^{m} \rightarrow S_{V}$ with $V$ maximal; that is, $V_{f}=\mathcal{H}_{m}^{p}$. Spherical minimal immersions corresponding to boundary points of $\mathcal{M}_{m}^{p}$ are said to be of boundary type.

In [1], Calabi proved that every full spherical minimal immersion $f: S^{2} \rightarrow S_{V}$ is congruent to the standard minimal immersion. In [3, 17], DoCarmo and Wallach showed that $\mathcal{M}_{m}^{p}$ can be identified with a compact convex body of an $S O(m+1)$ submodule $\mathcal{F}_{m}^{p}$ of the symmetric square $S^{2}\left(\mathcal{H}_{m}^{p}\right)$. They also proved that $\mathcal{F}_{m}^{p}$ is trivial for $p \leq 3$. By Calabi's result, $\mathcal{F}_{2}^{p}$ is trivial for $p \geq 1$, so that $\mathcal{F}_{m}^{p}$ can only be nontrivial for $m \geq 3$ and $p \geq 4$. They verified that this is indeed the case by giving a lower bound for $\mathcal{F}_{m}^{p}$ (in terms of irreducible subrepresentations). In [14], the author proved that the DoCarmo-Wallach lower bound for $\mathcal{F}_{m}^{p}$ is actually sharp. Using this, for $m \geq 3$ and $p \geq 4$, the complexification of $\mathcal{F}_{m}^{p}$ (denoted by the same symbol) can be decomposed as

$$
\mathcal{F}_{m}^{p} \cong \sum_{(a, b) \in \triangle_{2}^{p} ; a, b \text { even }} V_{m+1}^{(a, b, 0, \ldots, 0)}
$$

Here, $\triangle_{2}^{p} \subset \mathbf{R}^{2}$ denotes the closed convex triangle with vertices $(4,4),(p, p)$ and $(2(p-2), 4)$, and $V_{m+1}^{\left(u_{1}, \ldots, u_{d}\right)}, d=\operatorname{rank}(S O(m+1))=[|(m+1) / 2|]$, stands for the complex irreducible $S O(m+1)$-module with highest weight vector $\left(u_{1}, \ldots, u_{d}\right) \in$ $(\mathbf{Z} / 2)^{d}$ (relative to the standard maximal torus in $S O(m+1)$ ). For $m=3$, $V_{m+1}^{\left(u_{1}, \ldots, u_{d}\right)}$ actually means $V_{4}^{\left(u_{1}, u_{2}\right)} \oplus V_{4}^{\left(u_{1},-u_{2}\right)}$.

Disregarding conformality in the definition of a spherical minimal immersion, one arrives at the concept of an eigenmap. A map $f: S^{m} \rightarrow S_{V}$ is said to be a $p$-eigenmap if each component of $f$ is a spherical harmonic of order $p$. An eigenmap is harmonic in the sense of J.Eells and J.H.Sampson [4]; in fact, a harmonic map between spheres is an eigenmap iff it has constant energy density. Note also that 
an eigenmap is a spherical minimal immersion iff it is conformal, and, in this case, a $p$-eigenmap corresponds to a spherical minimal immersion of degree $p$.

In perfect analogy with spherical minimal immersions, the moduli space $\mathcal{L}_{m}^{p}$ of congruence classes of full p-eigenmaps $f: S^{m} \rightarrow S_{V}$ can be identified with a compact convex body of an $S O(m+1)$-submodule $\mathcal{E}_{m}^{p}$ of $S^{2}\left(\mathcal{H}_{m}^{p}\right)$. We have $\mathcal{F}_{m}^{p} \subset \mathcal{E}_{m}^{p}$ [3] and $\mathcal{M}_{m}^{p}=\mathcal{L}_{m}^{p} \cap \mathcal{F}_{m}^{p}$. Moreover, $\mathcal{E}_{m}^{p}$ is nontrivial iff $m \geq 3$ and $p \geq 2$; in fact, Calabi's result applies directly to this more general situation. In this case, the complexification of $\mathcal{E}_{m}^{p}$ decomposes as

$$
\mathcal{E}_{m}^{p} \cong \sum_{(a, b) \in \triangle_{1}^{p} ; a, b \text { even }} V_{m+1}^{(a, b, 0, \ldots, 0)}
$$

where $\triangle_{1}^{p} \subset \mathbf{R}^{2}$ is the closed convex triangle with vertices $(2,2),(p, p)$ and $(2(p-1), 2)$.

The condition of homothety (1), imposed on an eigenmap, can be viewed as a condition comparing the effect of the differential, the first fundamental form $\beta_{1}$, of $f$ with that of the standard minimal immersion $f_{p}$ :

$$
\left\langle\beta_{1}(f)(X), \beta_{1}(f)(Y)\right\rangle=\left\langle\beta_{1}\left(f_{p}\right)(X), \beta_{1}\left(f_{p}\right)(Y)\right\rangle,
$$

where $X, Y$ are vector fields on $S^{m}$. A spherical minimal immersion $f: S^{m} \rightarrow S_{V}$ of degree $p$ is said to be isotropic [8] with order of isotropy $k, 1 \leq k \leq p$, if this condition holds with the first fundamental forms replaced by the $l$-th fundamental forms for all $1 \leq l \leq k$. Isotropy of order $k$ essentially means that $f$ and $f_{p}$ have $k$-th order contact in the sense that, up to degree $k$, their osculating bundles are isomorphic. In [8], H.Gauchman and the author proved that, if $p \leq 2 k+1$, then a full isotropic minimal immersion of degree $p$ and order of isotropy $k$ is congruent to the standard minimal immersion $f_{m, p}$. Moreover, for $m \geq 4$ and $p \geq 2(k+1)$, the space $\mathcal{M}_{m}^{p ; k}$ of congruence classes of full isotropic minimal immersions of degree $p$ and order of isotropy $k$ can be identified by a compact convex body in the $S O(m+1)$ submodule $\mathcal{F}_{m}^{p ; k}$ of $S^{2}\left(\mathcal{H}_{m}^{p}\right)$ whose complexification decomposes as

$$
\mathcal{F}_{m}^{p ; k} \cong \sum_{(a, b) \in \triangle_{k+1}^{p} ; a, b \text { even }} V_{m+1}^{(a, b, 0, \ldots, 0)}
$$

where $\triangle_{k+1}^{p} \subset \mathbf{R}^{2}$ is the closed convex triangle with vertices $(2(k+1), 2(k+1))$, $(p, p)$ and $(2(p-k-1), 2(k+1))$. In particular, for $p$ even and $k=\frac{p}{2}-1$ maximal, $\mathcal{F}_{m}^{p, p / 2-1} \cong V_{m+1}^{(p, p, 0, \ldots, 0)}$ is irreducible. For $p$ odd and $k=\frac{(p-1)}{2}-1, \mathcal{F}_{m}^{p,(p-1) / 2-1} \cong$ $V_{m+1}^{(p-1, p-1,0, \ldots, 0)} \oplus V_{m+1}^{(p+1, p-1,0, \ldots, 0)}$ is the sum of two irreducible components.

We now proceed to describe the main construction. All results stated here will be proved in the forthcoming sections. Let $G \subset S O(m+1)$ be a closed subgroup with Lie algebra $\mathcal{G}$ and assume that $G$ acts transitively on $S^{m}$. For each $X \in \mathcal{G}$, we denote by $X^{*}$ the vector field induced by $X$ on $S^{m}$. Given a $p$-eigenmap $f: S^{m} \rightarrow S_{V}$, we define $\hat{f}: S^{m} \rightarrow V \otimes \mathcal{G}^{*}$ by

$$
\hat{f}(x)(X)=\lambda_{p}^{-1 / 2} X_{x}^{*}(f), \quad X \in \mathcal{G}, x \in S^{m} .
$$

Note that $\hat{f}$ may not be full even if $f$ is. To circumvent this minor technical difficulty, we usually restrict a non full map to the linear span of the image and denote the full restriction by the same symbol. The full $p$-eigenmap obtained from $f$ by applying ${ }^{\wedge} n$ times, $n \in \mathbf{N}$, to $f: S^{m} \rightarrow S_{V}$, will be denoted by $\hat{f}^{n}$. 
Theorem 1. (i) Given a p-eigenmap $f: S^{m} \rightarrow S_{V}$, the image of $\hat{f}$ is contained in the unit sphere of $V \otimes \mathcal{G}^{*}$, so that, by restriction, $\hat{f}: S^{m} \rightarrow S_{V \otimes \mathcal{G}^{*}}$ is a peigenmap. (ii) On the congruence classes, the correspondence $f \mapsto \hat{f}$ gives rise to a self-map of $\mathcal{L}_{m}^{p}$ which is the restriction of a symmetric linear map $\mathcal{A}_{m, p}: \mathcal{E}_{m}^{p} \rightarrow \mathcal{E}_{m}^{p}$. (iii) All eigenvalues of $\mathcal{A}_{m, p}$ are real and contained in $[-1,1]$. (iv) With respect to the $G$-module structure on $\mathcal{E}_{m}^{p}$ (by restriction), $\mathcal{A}_{m, p}$ is an endomorphism of $G$ modules. The eigenspace of $\mathcal{A}_{m, p}$ corresponding to the eigenvalue +1 is the fixed point set $\operatorname{Fix}_{G}\left(\mathcal{E}_{m}^{p}\right)$ of $G$ on $\mathcal{E}_{m}^{p}$ (whose intersection with $\mathcal{L}_{m}^{p}$ parametrizes the full $G$-equivariant p-eigenmaps $f: S^{m} \rightarrow S_{V}$ ). The eigenspace of $\mathcal{A}_{m, p}$ corresponding to the eigenvalue -1 is contained in the orthogonal complement of $\operatorname{Fix}_{G}\left(\mathcal{E}_{m}^{p}\right)$ in $\operatorname{Fix}_{[G, G]}\left(\mathcal{E}_{m}^{p}\right)$. In particular, -1 is not an eigenvalue if $G$ is semisimple.

By (2), $\mathcal{E}_{m}^{p}$ has multiplicity one decomposition into irreducible $S O(m+1)$ submodules. Since $\mathcal{A}_{m, p}$ is an endomorphism of $G$-modules, for $G=S O(m+1)$, it follows immediately that $\mathcal{A}_{m, p}$ maps $\mathcal{F}_{m}^{p}$ (and thereby $\mathcal{M}_{m}^{p}$ ) into itself. In the general case of a closed subgroup $G \subset S O(m+1)$ acting transitively on $S^{m}$, we have:

Theorem 2. If $f: S^{m} \rightarrow S_{V}$ is a spherical minimal immersion of degree $p$ then so is $\hat{f}$. Equivalently, $\mathcal{A}_{m, p}$ maps $\mathcal{M}_{m}^{p}$ into itself.

We will discuss two particular cases in detail. First, for $m=3$, we have the splitting $S O(4)=S U(2) \cdot S U(2)^{\prime}$ and, setting $G=S U(2)^{\prime}$, we will consider $\mathcal{A}_{3, p}$ acting on the linear slice $\operatorname{Fix}_{S U(2)}\left(\mathcal{L}_{3}^{p}\right)$ of $\mathcal{L}_{3}^{p}$ by the $S U(2)^{\prime}$-submodule $\operatorname{Fix}_{S U(2)}\left(\mathcal{E}_{3}^{p}\right)$ that parametrizes the full $S U(2)$-equivariant $p$-eigenmaps $f: S^{3} \rightarrow S_{V}$. In estimating the range dimensions of full $S U(2)$-equivariant $p$-eigenmaps $f: S^{3} \rightarrow S_{V}$, the following two facts will be important in the sequel: (1) $V$ is an $S U(2)$-submodule of $\mathcal{H}_{3}^{p}$ (via $V^{*} \cong V_{f} \subset \mathcal{H}_{3}^{p}$ ) and therefore [2] $\operatorname{dim} V$ is divisible by $p+1$; (2) The number of irreducible $S U(2)^{\prime}$-submodules of $\operatorname{Fix}_{S U(2)}\left(\mathcal{E}_{3}^{p}\right)$ is $[|p / 2|]$ and those of $\operatorname{Fix}_{S U(2)}\left(\mathcal{F}_{3}^{p}\right)$ is $[|p / 2|]-1$. (This was established in [2] by a 'heuristic argument' and proved in [15].)

Second, for $m \geq 4$ and $p$ even, setting $G=S O(m+1)$, we will consider $\mathcal{A}_{m, p}$ acting on $\mathcal{F}_{m}^{p ; p / 2-1} \cong V_{m+1}^{(p, p, 0, \ldots, 0)}$ whose slice with $\mathcal{M}_{m}^{p}$ parametrizes the full isotropic minimal immersions $f: S^{m} \rightarrow S_{V}$ of degree $p$ and order of isotropy $p / 2-1$. Similarly, for $p$ odd, $\mathcal{A}_{m, p}$ acts on the sum $\mathcal{F}_{m}^{p,(p-1) / 2-1} \cong V_{m+1}^{(p-1, p-1,0, \ldots, 0)} \oplus$ $V_{m+1}^{(p+1, p-1,0, \ldots, 0)}$ whose slice with $\mathcal{M}_{m}^{p}$ parametrizes the full isotropic minimal immersions $f: S^{m} \rightarrow S_{V}$ of degree $p$ and order of isotropy $(p-1) / 2-1$.

Recall the triangle $\triangle_{1}^{p}$ in (2) whose even coordinate points give the nonzero components of the highest weights of representations that occur in $\mathcal{E}_{m}^{p}$. For $p \leq q$, we have $\triangle_{1}^{p} \subset \triangle_{1}^{q}$, so that $\mathcal{E}_{m}^{p}$ is an $S O(m+1)$-submodule of $\mathcal{E}_{m}^{q}$. The following result shows that the eigenvalues of $\mathcal{A}_{m, p}$ on $\mathcal{E}_{m}^{p}$ determine the eigenvalues of $\mathcal{A}_{m, q}$ on $\mathcal{E}_{m}^{p} \subset \mathcal{E}_{m}^{q}$.

Theorem 3. Let $\lambda$ be an eigenvalue of $\mathcal{A}_{m, p}$ on an irreducible component of $\mathcal{E}_{m}^{p}$. Then, for $p \leq q$, the eigenvalue of $\mathcal{A}_{m, q}$ on the same irreducible component of $\mathcal{E}_{m}^{q}$ is

$$
\Lambda(\lambda, p, q)=1-(1-\lambda) \frac{\lambda_{p}}{\lambda_{q}},
$$

where $\lambda_{p}=p(p+m-1)$ is the $p$-th eigenvalue of $\triangle^{S^{m}}$. 
Remark 1.

$$
\Lambda(\Lambda(\lambda, p, q), q, r)=\Lambda(\lambda, p, r), \quad p \leq q \leq r .
$$

In particular, it is enough to prove Theorem 3 for $q=p+1$.

Remark 2. If $\lambda \geq-1$ then, given $p \geq 2$, there is a least $q \leq 2 p$ such that $\Lambda(\lambda, p, q)>$ 0 . In particular, by Theorems 1 and 3 , all eigenvalues of $\mathcal{A}_{m, 2 p}$ on $\mathcal{E}_{m}^{p} \subset \mathcal{E}_{m}^{2 p}$ are positive.

Let $m=3$. The eigenvalue of $\mathcal{A}_{3, p}$ on the irreducible $S U(2)^{\prime}$-submodule $\operatorname{Fix}_{S U(2)}\left(\mathcal{E}_{3}^{2}\right)$ of $\mathcal{E}_{3}^{p}, p \geq 2$, is $1-12 /(p(p+2))$. This will follow from Theorem 3 by computations for $p=2$; in fact, the eigenvalue of $\mathcal{A}_{3,2}$ on $\operatorname{Fix}_{S U(2)}\left(\mathcal{E}_{3}^{2}\right)$ is $-1 / 2$. Similarly, the eigenvalue of $\mathcal{A}_{3, p}$ on the irreducible $S U(2)^{\prime}$-submodule $\operatorname{Fix}_{S U(2)}\left(\mathcal{F}_{3}^{4}\right)$ of $\mathcal{F}_{3}^{p}, p \geq 4$, is $1-40 /(p(p+2))$. These computations will be carried out in the last section.

Assume, from now on, that $G$ is semisimple. By Theorem $1, \mathcal{A}_{m, p}$ is the identity map on $\operatorname{Fix}_{G}\left(\mathcal{E}_{m}^{p}\right)$ and a contraction on the orthogonal complement of $\operatorname{Fix}_{G}\left(\mathcal{E}_{m}^{p}\right)$ in $\mathcal{E}_{m}^{p}$. Given a full p-eigenmap $f: S^{m} \rightarrow S_{V}$, we define the critical exponent $e(f)$ of $f$ as follows: If the congruence class $C$ of $f$ is in the interior of $\mathcal{L}_{m}^{p}$, then we define $e(f)$ to be zero. If $C$ is on the boundary of $\mathcal{L}_{m}^{p}$, then we consider the sequence $C, \mathcal{A}_{m, p}(C),\left(\mathcal{A}_{m, p}\right)^{2}(C), \ldots$ If all these points are on the boundary of $\mathcal{L}_{m}^{p}$, then we define $e(f)$ to be infinite. Otherwise, we define $e(f)$ to be the first integer $n \geq 0$ such that $\left(\mathcal{A}_{m, p}\right)^{n}(C)$ is in the interior of $\mathcal{L}_{m}^{p}$. If $C$ is orthogonal to $\operatorname{Fix}_{G}\left(\mathcal{E}_{m}^{p}\right)$, then the critical exponent $e(f)$ is clearly finite.

Assume that $f: S^{m} \rightarrow S_{V}$ is a full $p$-eigenmap with $2 \leq e(f)<\infty$. The range of $\hat{f}^{e(f)}$ is $V \otimes \mathcal{U}^{e(f)}(\mathcal{G})^{*}$, where $\mathcal{U}(G)$ is the universal enveloping algebra of $\mathcal{G}$, and $\mathcal{U}^{d}(\mathcal{G})$ is the linear subspace of elements of degree $\leq d$. This is because the components of $\hat{f}^{d}$ are obtained by applying monomials of degree $\leq d$ of infinitesimal isometries to the components of $f$. By the definition of $e(f),\left\langle\hat{f}^{e}(f)\right\rangle$ is an interior point of $\mathcal{L}^{p}$, so that the components of $\hat{f}^{e(f)}$ span $\mathcal{H}_{m}^{p}$. We thus have

$$
\operatorname{dim} \mathcal{H}_{m}^{p} \leq \operatorname{dim} V \cdot \operatorname{dim}\left(\mathcal{U}^{e(f)}(\mathcal{G})\right)
$$

and we obtain the lower estimate

$$
\operatorname{dim} V \geq \frac{\operatorname{dim} \mathcal{H}_{m}^{p}}{\operatorname{dim}\left(\mathcal{U}^{e(f)}(\mathcal{G})\right)}=\frac{\operatorname{dim} \mathcal{H}_{m}^{p}}{\left(\begin{array}{c}
\operatorname{dim} G+e(f) \\
e(f)
\end{array}\right)-1} .
$$

For example, consider the possible range dimensions of full cubic $S U(2)$-equivariant eigenmaps $f: S^{3} \rightarrow S_{V}$. As noted above, $\mathcal{A}_{3,3}$ acting on the irreducible $S U(2)^{\prime}$ module $\operatorname{Fix}_{S U(2)}\left(\mathcal{E}_{3}^{3}\right)$ has the single positive eigenvalue $(1-12 /(3 \cdot 5))$. Thus $\mathcal{A}_{3,3}$ maps the entire boundary of $\mathcal{L}_{3}^{3}$ into the interior of $\mathcal{L}_{3}^{3}$. We obtain $e(f) \leq 1$. By (6), we have $\operatorname{dim} V \geq \operatorname{dim} \mathcal{H}_{3}^{3} / 3=16 / 3$. On the other hand, $\operatorname{dim} V$ is divisible by 4 and we obtain $\operatorname{dim} V \geq 8$.

The following example (due to H.Gauchman) shows that an arbitrary large power of a linear contraction $\mathcal{A}$ with distinct eigenvalues may map boundary points of an $\mathcal{A}$-invariant compact convex body $\mathcal{L}$ to boundary points.

Example 1. For $n \in \mathbf{N}$, let $\mathcal{L} \subset \mathbf{R}^{2}$ be the convex polygon with vertices $(1, \pm 1)$, $\left(1 / 3^{k}, \pm 1 / 2^{k}\right), k=1, \ldots, n$, and $\left(-1 / 3^{n}, 0\right)$, and let $\mathcal{A}$ be the diagonal matrix with diagonal entries $1 / 3$ and $1 / 2$. Clearly, $\mathcal{A}, \mathcal{A}^{2}, \ldots, \mathcal{A}^{n}$ map some points of the boundary of $\mathcal{L}$ to boundary points of $\mathcal{L}$ and $\mathcal{A}^{n+1}$ maps the entire $\mathcal{L}$ into its interior. 
The lower bound in (6) can be significantly improved when the congruence class corresponding to the eigenmap in question is contained in a limited number of irreducible components of $\mathcal{E}_{m}^{p}$. For example, $\mathcal{A}_{m, p}$ restricted to an irreducible component has a unique eigenvalue so that $\left(\mathcal{A}_{m, p}\right)^{2}$ maps the entire slice of $\mathcal{L}_{m}^{p}$ by this component into the interior of $\mathcal{L}_{m}^{p}$. A more careful argument will establish the main result of this paper:

Theorem 4. Let $G$ be semisimple and assume that the congruence class of a full p-eigenmap $f: S^{m} \rightarrow S_{V}$ is contained in an $\mathcal{A}_{m, p}$-invariant $G$-submodule $\mathcal{V}$ of $\mathcal{E}_{m}^{p}$ orthogonal to $\operatorname{Fix}_{G}\left(\mathcal{E}_{m}^{p}\right)$. Let d be the number of distinct eigenvalues of $\mathcal{A}_{m, p}$ on $\mathcal{V}$. If $d=1$, then $e(f) \leq 2$ and we have

$$
\operatorname{dim} V \geq \frac{\operatorname{dim} \mathcal{H}_{m}^{p}}{1+\operatorname{dim} G}
$$

If $d \geq 2$, then $e(f) \leq d$ so that we have

$$
\operatorname{dim} V \geq \frac{\operatorname{dim} \mathcal{H}_{m}^{p}}{\operatorname{dim} \mathcal{U}^{d}(\mathcal{G})}=\frac{\operatorname{dim} \mathcal{H}_{m}^{p}}{\left(\begin{array}{c}
\operatorname{dim} G+d \\
d
\end{array}\right)-1} .
$$

Remark. The eigenvalues of $\mathcal{A}_{m, p}$ on $\mathcal{E}_{m}^{p}$ can be computed, at least theoretically, since $\mathcal{E}_{m}^{p}$ is a factor of $S^{2}\left(\mathcal{H}_{m}^{p}\right)$.

For quartic and quintic $S U(2)$-equivariant spherical immersions, we have $d=1$, and we recover the lower estimates of Moore [10] and DeTurck-Ziller [2]:

Corollary 1. Let $f: S^{3} \rightarrow S_{V}$ be an $S U(2)$-equivariant spherical minimal immersion of degree $p$. (a) If $p=4$, then $\operatorname{dim} V \geq 10$. (b) If $p=5$, then $\operatorname{dim} V \geq 12$.

It is revealing to look at the connection between the existence of the $S U(2)$ equivariant spherical minimal immersion $f: S^{2} \rightarrow S^{6}$ of degree 6 mentioned above and the geometry of $\mathcal{M}_{3}^{6}$. Indeed, the $S U(2)^{\prime}$-module $\operatorname{Fix}_{S U(2)}\left(\mathcal{F}_{3}^{6}\right)$ has two irreducible components: $\operatorname{Fix}_{S U(2)}\left(\mathcal{F}_{3}^{4}\right)$ and its complement. As noted above, the eigenvalue of $\mathcal{A}_{3,6}$ on $\operatorname{Fix}_{S U(2)}\left(\mathcal{F}_{3}^{4}\right)$ is positive $(1-40 /(6 \cdot 8))$. Let $f: S^{3} \rightarrow S_{V}$ be a full $S U(2)$-equivariant minimal immersion of degree 6 . If the congruence class of $f$ belongs to $\operatorname{Fix}_{S U(2)}\left(\mathcal{F}_{3}^{4}\right)$, then $e(f) \leq 1$ so that $\operatorname{dim} V \geq 49 / 3$. Since $7 \mid \operatorname{dim} V$, we obtain $\operatorname{dim} V \geq 21$. If the congruence class of $f$ belongs to the complement of $\operatorname{Fix}_{S U(2)}\left(\mathcal{F}_{3}^{4}\right)$, then, by Theorem $4, \operatorname{dim} V \geq 49 /(1+3)$, so that $\operatorname{dim} V \geq 14$. We conclude that the congruence class of the minimal example $f: S^{3} \rightarrow S^{6}$ cannot be contained in the convex hull of these two irreducible components. (It is also clear that $\mathcal{A}_{3,6}$ has two distinct eigenvalues on $\operatorname{Fix}_{S U(2)}\left(\mathcal{F}_{3}^{6}\right)$ and, by Theorem 4 , $e(f)=2$.) This and many other examples [2] show that $\mathcal{M}_{m}^{p}, p \geq 6$, is not the convex hull of its slices by irreducible components of $\mathcal{F}_{m}^{p}$. This is in sharp contrast with a result of Ziller and the author [15] that asserts that $\mathcal{M}_{3}^{4}$ is the convex hull of the slices $\operatorname{Fix}_{S U(2)}\left(\mathcal{M}_{3}^{4}\right)$ and $\operatorname{Fix}_{S U(2)^{\prime}}\left(\mathcal{M}_{3}^{4}\right)$ that correspond to $S U(2)$ and $S U(2)^{\prime}$-equivariant quartic spherical minimal immersions.

Remark. The example $f: S^{3} \rightarrow S^{6}$ above can easily be generalized. In fact, for $p \geq 6$ even, the 'equivariant construction' [2] applied to an irreducible $S U(2)$ submodule of $\mathcal{H}_{3}^{p}$ gives spherical minimal immersions $f: S^{3} \rightarrow S^{p}$ of degree $p$ (since the system of equations for minimality is solvable for $p \geq 6$ ). For $G=S U(2)^{\prime}$, the lower estimate $(6)\left(\operatorname{dim} \mathcal{H}_{3}^{p}=(p+1)^{2}\right)$ and Theorem 4 give

$$
\ln (p+1) / \ln 3 \leq e(f) \leq[|p / 2|]-1 .
$$


Corollary 2. Let $m \geq 4$ and $p$ even. Let $f: S^{m} \rightarrow S_{V}$ be an isotropic minimal immersion of degree $p$ and order of isotropy $p / 2-1$. Then, we have

$$
\operatorname{dim} V \geq \frac{\operatorname{dim} \mathcal{H}_{m}^{p}}{1+\operatorname{dim} S O(m+1)}=\frac{2(m+2 p-1)(m+p-2) !}{p !(m-1) !(m(m+1)+2)} .
$$

Corollary 2 is a direct application of Theorem 4 since the stated full isotropic minimal immersions are parametrized by the slice of $\mathcal{M}_{m}^{p}$ with the $S O(m+1)$ submodule $\mathcal{F}_{m}^{p ; p / 2-1}$ of $\mathcal{F}_{m}^{p}$ which, by (3), is irreducible, and isomorphic with $V_{m+1}^{(p, p, 0, \ldots, 0)}$. Although they are abundant, no explicit construction is known for minimal immersions of degree $\geq 6$ and order of isotropy $\geq 2$. By Corollary 2, the minimum range for an isotropic minimal immersion of degree 6 and order of isotropy 2 from $S^{4}$ is $S^{12}$ and from $S^{5}$ is $S^{20}$.

Corollary 3. Let $m \geq 4$ and $p$ odd. Let $f: S^{m} \rightarrow S_{V}$ be an isotropic minimal immersion of degree $p$ and order of isotropy $(p-1) / 2-1$. Then, we have

$$
\operatorname{dim} V \geq \frac{\operatorname{dim} \mathcal{H}_{m}^{p}}{(\operatorname{dim} S O(m+1))^{2}}=\frac{4(m+2 p-1)(m+p-2) !}{p ! m(m+1)(m+1) !} .
$$

Remark. If $f: S^{m} \rightarrow S_{V}, m \geq 3$, is a full isotropic minimal immersion of degree $p$ and order of isotropy $k$, then the $l$-th osculating bundle, $l=1, \ldots, k$, has fibres isomorphic with $\mathcal{H}_{m-1}^{l}$ [8]. Thus, we have

$$
\operatorname{dim} V \geq \operatorname{dim}\left(\mathcal{H}_{m-1}^{0} \oplus \ldots \oplus \mathcal{H}_{m-1}^{k}\right)=\operatorname{dim} \mathcal{H}_{m}^{k} .
$$

In particular, if $p$ is even and $k=p / 2-1$, then the weaker lower estimate $\operatorname{dim} V \geq$ $\operatorname{dim} \mathcal{H}_{m}^{p / 2-1}$ follows.

Since spherical minimal immersions are isotropic of order 1 , a special case of Corollary $2(p=4)$ is the following:

Corollary 4. Let $m \geq 4$ and $f: S^{m} \rightarrow S_{V}$ a quartic minimal immersion. Then, we have

$$
\operatorname{dim} V \geq \frac{m(m+1)(m+2)(m+7)}{12(m(m+1)+2)}
$$

Note that the lower bound here is $\mathcal{O}\left(m^{2}\right)$; a significant improvement (for $m \geq 15$ ) of Moore's (linear) lower bound in the quartic case.

For isotropic minimal immersions, the first notable improvement in the lower estimate on the range dimension is $\mathcal{O}\left(\mathrm{m}^{3}\right)$ in degree 7 :

Corollary 5. Let $m \geq 4$ and $f: S^{m} \rightarrow S_{V}$ be a minimal immersion of degree 7 and order of isotropy 2. Then, we have

$$
\operatorname{dim} V \geq \frac{(m+2)(m+3)(m+4)(m+5)(m+13)}{1260 m(m+1)} .
$$

\section{ACKNOWLEDGEMENT}

The author wishes to thank the referee for the careful reading of the manuscript and for suggesting various improvements. 


\section{InFinitesimal Rotations of AN EigenMaP}

A spherical harmonic $h$ of order $p$ on $S^{m}$; that is, an eigenfunction of $\triangle^{S^{m}}$ with eigenvalue $\lambda_{p}$, is the restriction of a harmonic $p$-homogeneous polynomial in $m+1$ variables [16]. In what follows, we identify these two representations of spherical harmonics. In particular, a $p$-eigenmap $f: S^{m} \rightarrow S_{V}$ is the restriction of a harmonic p-homogeneous polynomial map $f: \mathbf{R}^{m+1} \rightarrow V$ to the respective spheres.

Let $G \subset S O(m+1)$ be a closed subgroup acting transitively on $S^{m}$. (For classification results, cf. [18].) Let $\mathcal{G}$ be the Lie algebra of $G$ and endow $\mathcal{G}$ with the bi-invariant Riemannian metric which induces the standard (curvature one) Riemannian metric on $S^{m}$. Given an orthonormal basis $\left\{E_{i}\right\}_{i=1}^{s} \subset \mathcal{G}$, we have $\triangle^{S^{m}}=-\sum_{i=1}^{s}\left(E_{i}^{*}\right)^{2}[17]$. Let $f: S^{m} \rightarrow S_{V}$ be a $p$-eigenmap. In terms of the dual basis $\left\{\phi_{i}\right\}_{i=1}^{s} \subset \mathcal{G}^{*}$, the map $\hat{f}: S^{m} \rightarrow V \otimes \mathcal{G}^{*}$ in (4) is given by

$$
\hat{f}=\lambda_{p}^{-1 / 2} \sum_{i=1}^{s} E_{i}^{*}(f) \otimes \phi_{i} .
$$

Since each $X^{*}, X \in \mathcal{G}$, is an infinitesimal isometry on $S^{m}$, the components of $\hat{f}$ are spherical harmonics of order $p$ on $S^{m}$. To prove that $\hat{f}$ maps into the unit sphere of $V \otimes \mathcal{G}^{*}$, we first note that

$$
\begin{aligned}
\triangle^{S^{m}}|f|^{2} & =2\left\langle\triangle^{S^{m}} f, f\right\rangle-2 \sum_{i=1}^{s}\left|E_{i}^{*}(f)\right|^{2} \\
& =2 \lambda_{p}|f|^{2}-2 \sum_{i=1}^{s}\left|E_{i}^{*}(f)\right|^{2}
\end{aligned}
$$

and this is zero since $|f|^{2}=1$ on $S^{m}$. Thus, we have

$$
|\hat{f}|^{2}=1 / \lambda_{p} \sum_{i=1}^{s}\left|E_{i}^{*}(f)\right|^{2}=|f|^{2}=1 .
$$

The first statement (i) of Theorem 1 follows.

We now recall the construction of the moduli space $\mathcal{L}_{m}^{p}$. Unless relevant, we suppress $m$ from the notation and write $\mathcal{L}^{p}$, etc. Let $\mathcal{H}^{p}$ denote the vector space of spherical harmonics of order $p$ on $S^{m}$ with $L_{2}$-scalar product

$$
\left\langle h, h^{\prime}\right\rangle=\frac{n(p)+1}{\operatorname{vol}\left(S^{m}\right)} \int_{S^{m}} h h^{\prime} v_{S^{m}}, \quad h, h^{\prime} \in \mathcal{H}^{p},
$$

where

$$
\operatorname{dim} \mathcal{H}^{p}=n(p)+1=(m+2 p-1) \frac{(m+p-2) !}{p !(m-1) !} .
$$

In terms of an orthonormal basis $\left\{f_{p}^{j}\right\}_{j=0}^{n(p)} \subset \mathcal{H}^{p}$, the standard minimal immersion $f_{p}: S^{m} \rightarrow S_{\mathcal{H}^{p}}$ is given by

$$
f_{p}(x)=\sum_{j=0}^{n(p)} f_{p}^{j}(x) f_{p}^{j} .
$$

The DoCarmo-Wallach parametrization of $\mathcal{L}^{p}[3]$ is given as follows: Given a full p-eigenmap $f: S^{m} \rightarrow S_{V}$, the space of components $V_{f}=\left\{\phi \circ f \mid \phi \in V^{*}\right\}\left(\cong V^{*}\right)$ is contained in $\mathcal{H}^{p}$. Thus, there exists a linear map $A: \mathcal{H}^{p} \rightarrow V$ such that $f=A \circ f_{p}$. Notice that $A$ is uniquely determined and, since it is onto, $\operatorname{rank} A=\operatorname{dim} V$. We now 
associate to (the congruence class of) $f$ the symmetric endomorphism $\langle f\rangle=A^{\top} A-I$ of $\mathcal{H}^{p}$; an element of $S^{2}\left(\mathcal{H}^{p}\right)$. The image of the correspondence $f \mapsto\langle f\rangle$ is the moduli space $\mathcal{L}^{p}$. Its linear span $\mathcal{E}^{p}$ is given by

$$
\mathcal{E}^{p}=\left\{C \in S^{2}\left(\mathcal{H}^{p}\right) \mid\langle C, f(x) \odot f(x)\rangle=\langle C f(x), f(x)\rangle=0, x \in S^{m}\right\},
$$

where $S^{2}\left(\mathcal{H}^{p}\right)$ carries the usual scalar product defined by $\left\langle C, C^{\prime}\right\rangle=\operatorname{trace}\left(C C^{\prime}\right)$, $C, C^{\prime} \in S^{2}\left(\mathcal{H}^{p}\right)$, and $\odot$ denotes the symmetric tensor product. Since $A^{\top} A$ is positive semidefinite for all $A$, we have

$$
\mathcal{L}^{p}=\left\{C \in \mathcal{E}_{m}^{p} \mid C+I \geq 0\right\} .
$$

The interior of $\mathcal{L}^{p}$ is defined replacing $\geq$ by sharp inequality, so that the interior points correspond to those full $p$-eigenmaps $f: S^{m} \rightarrow S_{V}$ for which $V \cong V_{f}^{*}$ is maximal. This follows since $\operatorname{rank}\left(A^{\top} A\right)=\operatorname{rank} A=\operatorname{dim} V$. A full $p$-eigenmap $f: S^{m} \rightarrow S_{V}$ is said to be of boundary type if $\langle f\rangle \in \partial \mathcal{L}^{p}$; that is, $\operatorname{dim} V<\operatorname{dim} \mathcal{H}^{p}$. The following simple lemma follows almost directly from the definitions (cf. [15]).

Connecting Lemma. Let $f_{1}: S^{m} \rightarrow S_{V_{1}}$ and $f_{2}: S^{m} \rightarrow S_{V_{2}}$ be incongruent full p-eigenmaps. Let $c_{1}, c_{2}>0$ with $c_{1}+c_{2}=1$. Then the point

$$
c_{1}\left\langle f_{1}\right\rangle+c_{2}\left\langle f_{2}\right\rangle \in \mathcal{L}^{p}
$$

on the segment connecting $\left\langle f_{1}\right\rangle$ and $\left\langle f_{2}\right\rangle$ is represented by the p-eigenmap $f: S^{m} \rightarrow$ $S_{V}, V=V_{1} \times V_{2}$, defined by $f=\left(\sqrt{c_{1}} f_{1}, \sqrt{c_{2}} f_{2}\right)$ and made full. In particular, for the spaces of components, we have

$$
V_{f}=V_{f_{1}}+V_{f_{2}}
$$

To realize the correspondence $f \mapsto \hat{f}$ on the moduli space $\mathcal{L}^{p}$, we first introduce the linear map

$$
\alpha_{p}: \mathcal{H}^{p} \rightarrow \mathcal{H}^{p} \otimes \mathcal{G}^{*}
$$

defined by

$$
\alpha_{p}(h)(X)=\lambda_{p}^{-1 / 2} X^{*}(h), \quad h \in \mathcal{H}^{p}, X \in \mathcal{G} .
$$

Lemma 1. We have

$$
\alpha_{p}^{\top} \circ \alpha_{p}=I .
$$

In particular, $\alpha_{p}: \mathcal{H}^{p} \rightarrow \mathcal{H}^{p} \otimes \mathcal{G}^{*}$ is a linear isometric imbedding.

Proof. The vector field $X^{*}, X \in \mathcal{G}$, is an infinitesimal isometry on $S^{m}$, so that its action on $\mathcal{H}^{p}$ is skew-symmetric. It follows that the transpose $\alpha_{p}^{\top}$ is given by

$$
\alpha_{p}^{\top}\left(h \otimes \phi_{i}\right)=-\lambda_{p}^{-1 / 2} E_{i}^{*}(h), \quad h \in \mathcal{H}^{p}, i=1, \ldots, s .
$$

We use this to compute

$$
\begin{aligned}
\left(\alpha_{p}^{\top} \circ \alpha_{p}\right)(h) & =\alpha_{p}^{\top}\left(\lambda_{p}^{-1 / 2} \sum_{i=1}^{s} E_{i}^{*}(h) \otimes \phi_{i}\right) \\
& =-1 / \lambda_{p} \sum_{i=1}^{s}\left(E_{i}^{*}\right)^{2} h=1 / \lambda_{p} \triangle^{S^{m}} h=h .
\end{aligned}
$$

The next lemma asserts that $\hat{f}_{p}$, made full, is congruent to $f_{p}$.

Lemma 2. For $x \in S^{m}$, we have

$$
\hat{f}_{p}(x)=\alpha_{p}\left(f_{p}(x)\right) .
$$


Proof. We have

$$
\begin{aligned}
\hat{f}_{p}(x) & =\lambda_{p}^{-1 / 2} \sum_{i=1}^{s} \sum_{j=0}^{n(p)} f_{p}^{j}(x) E_{i}^{*}\left(f_{p}^{j}\right) \otimes \phi_{i} \\
& =\sum_{j=0}^{n(p)} f_{p}^{j}(x) \alpha_{p}\left(f_{p}^{j}\right)=\alpha_{p}\left(f_{p}(x)\right) .
\end{aligned}
$$

Let $f: S^{m} \rightarrow S_{V}$ be a full $p$-eigenmap with $\langle f\rangle=A^{\top} A-I \in \mathcal{L}^{p}$, where $A: \mathcal{H}^{p} \rightarrow V$ is a linear map with $f=A \circ f_{p}$. We have

$$
\hat{f}=(A \otimes I) \circ \hat{f}_{p}=(A \otimes I) \circ \alpha_{p} \circ f_{p} .
$$

Using Lemma 1, we compute

$$
\begin{aligned}
\langle\hat{f}\rangle & =\alpha_{p}^{\top}\left(A^{\top} \otimes I\right)(A \otimes I) \alpha_{p}-I \\
& =\alpha_{p}^{\top}\left(\left(A^{\top} A-I\right) \otimes I\right) \alpha_{p} \\
& =\alpha_{p}^{\top}(\langle f\rangle \otimes I) \alpha_{p} .
\end{aligned}
$$

In view of this, we define the linear map

$$
\mathcal{A}_{p}: S^{2}\left(\mathcal{H}^{p}\right) \rightarrow S^{2}\left(\mathcal{H}^{p}\right)
$$

by

$$
\mathcal{A}_{p}(C)=\alpha_{p}^{\top} \circ(C \otimes I) \circ \alpha_{p}, \quad C \in S^{2}\left(\mathcal{H}^{p}\right) .
$$

The computation above implies that $\mathcal{A}_{p}(\langle f\rangle)=\langle\hat{f}\rangle$; in particular, $\mathcal{L}^{p}$ is $\mathcal{A}_{p^{-}}$ invariant. The linear span $\mathcal{E}^{p}$ of $\mathcal{L}^{p}$ is also $\mathcal{A}_{p}$-invariant since $\mathcal{A}_{p}$ is linear. The second statement (ii) of Theorem 1 will follow if we prove the following:

Lemma 3. $\mathcal{A}_{p}: S^{2}\left(\mathcal{H}^{p}\right) \rightarrow S^{2}\left(\mathcal{H}^{p}\right)$ is symmetric.

Proof. Given $C, C^{\prime} \in S^{2}\left(\mathcal{H}^{p}\right)$, we compute

$$
\begin{aligned}
\left\langle\mathcal{A}_{p}(C), C^{\prime}\right\rangle & =\sum_{j=0}^{n(p)}\left\langle\mathcal{A}_{p}(C)\left(f_{p}^{j}\right), C^{\prime}\left(f_{p}^{j}\right)\right\rangle \\
& =\sum_{j=0}^{n(p)}\left\langle(C \otimes I) \alpha_{p}\left(f_{p}^{j}\right), \alpha_{p}\left(C^{\prime}\left(f_{p}^{j}\right)\right)\right\rangle \\
& =1 / \lambda_{p} \sum_{i=1}^{s} \sum_{j=0}^{n(p)}\left\langle C\left(E_{i}^{*}\left(f_{p}^{j}\right)\right), E_{i}^{*}\left(C^{\prime}\left(f_{p}^{j}\right)\right)\right\rangle \\
& =-1 / \lambda_{p} \sum_{i=1}^{s} \sum_{l=0}^{n(p)}\left\langle C\left(f_{p}^{l}\right), E_{i}^{*}\left(C^{\prime}\left(E_{i}^{*}\left(f_{p}^{l}\right)\right)\right)\right\rangle \\
& =1 / \lambda_{p} \sum_{i=1}^{s} \sum_{l=0}^{n(p)}\left\langle E_{i}^{*} C\left(f_{p}^{l}\right), C^{\prime}\left(E_{i}^{*}\left(f_{p}^{l}\right)\right)\right\rangle \\
& =\left\langle C, \mathcal{A}_{p}\left(C^{\prime}\right)\right\rangle,
\end{aligned}
$$

where we used the fact that infinitesimal isometries are skew on $\mathcal{H}^{p}$ and replaced $E_{i}^{*}\left(f_{p}^{j}\right)$ with $-\sum_{l=0}^{n(p)}\left\langle f_{p}^{j}, E_{i}^{*}\left(f_{p}^{l}\right)\right\rangle f_{p}^{l}$. The lemma follows. 
Since $\mathcal{A}_{p}$ is symmetric on $S^{2}\left(\mathcal{H}^{p}\right)$, it is diagonalizable and has real eigenvalues. Since $\mathcal{E}^{p}$ is $\mathcal{A}_{p}$-invariant, the same holds for the action of $\mathcal{A}_{p}$ on $\mathcal{E}^{p}$. Moreover, since $\mathcal{L}^{p} \subset \mathcal{E}^{p}$ is compact and $\mathcal{A}^{p}$-invariant, all eigenvalues of $\mathcal{A}_{p} \mid \mathcal{E}^{p}$ are in $[-1,1]$. The third statement (iii) of Theorem 1 follows.

Precomposition with the inverse of isometries of the orthogonal group $S O(m+1)$ defines an (orthogonal) action on $\mathcal{H}^{p}$; that is, we set $g \cdot h=h \circ g^{-1}, g \in S O(m+1)$, $h \in \mathcal{H}^{p}$. This action extends to the symmetric square $S^{2}\left(\mathcal{H}^{p}\right)$. The linear subspaces $\left(\mathcal{F}^{p ; k} \subset\right) \mathcal{F}^{p} \subset \mathcal{E}^{p} \subset S^{2}\left(\mathcal{H}^{p}\right)$ are $S O(m+1)$-submodules. In fact, if $f: S^{m} \rightarrow S_{V}$ is a full $p$-eigenmap then $g \cdot\langle f\rangle=\left\langle f \circ g^{-1}\right\rangle, g \in S O(m+1)$.

Let $G \subset S O(m+1)$ be a closed subgroup. The linear slice $\operatorname{Fix}_{G}\left(\mathcal{L}^{p}\right)$ parametrizes the (congruence classes of) full $p$-eigenmaps that are $G$-equivariant; the homomorphism $\rho: G \rightarrow S O(V)$ corresponding to $G$-equivariance of $f: S^{m} \rightarrow S_{V}$ depends on $f$. We have

$$
f \circ g=\rho(g) \circ f, \quad g \in G .
$$

From now on, let $G \subset S O(m+1)$ be a closed subgroup acting transitively on $S^{m}$ and let $\mathcal{G}$ be the Lie algebra of $G$. We define $\alpha_{p}: \mathcal{H}^{p} \rightarrow \mathcal{H}^{p} \otimes \mathcal{G}^{*}$ as above. For $g \in G, h \in \mathcal{H}^{p}$ and $X \in \mathcal{G}$, we have

$$
\begin{aligned}
\alpha_{p}(g \cdot h)(X) & =\lambda_{p}^{-1 / 2} X^{*}\left(h \circ g^{-1}\right) \\
& =\lambda_{p}^{-1 / 2}\left(g^{-1}\right)_{*} X^{*}(h) \\
& =\left(\lambda_{p}\right)^{-1 / 2}\left(\operatorname{ad}\left(g^{-1}\right) X\right)^{*}(h) \circ g^{-1} \\
& =g \cdot \alpha_{p}(h)\left(\operatorname{ad}\left(g^{-1}\right) X\right) .
\end{aligned}
$$

Here $\left(g^{-1}\right)_{*}$ denotes the differential of $g^{-1}$ considered as a self-map of $S^{m}$. This shows that $\alpha_{p}$ is a homomorphism of $G$-modules, where the module structure on $\mathcal{H}^{p}$ is given by restriction, and on $\mathcal{G}^{*}$ by the adjoint representation. As an immediate consequence, we also see that $\mathcal{A}_{p}$ is an endomorphism of the $G$-module $S^{2}\left(\mathcal{H}^{p}\right)$.

Lemma 4. $\operatorname{Fix}_{G}\left(\mathcal{E}^{p}\right)$ is equal to the +1 eigenspace of $\mathcal{A}_{p}$.

Proof. We first show that $\mathcal{A}_{p}$ is identity on $\operatorname{Fix}_{G}\left(\mathcal{E}^{p}\right)$. Being a homomorphism of $G$-modules, $\mathcal{A}_{p}$ leaves $\operatorname{Fix}_{G}\left(\mathcal{E}^{p}\right)$ invariant. By Lemma 3 , it remains to show that all eigenvalues of $\mathcal{A}_{p}$ on $\operatorname{Fix}_{G}\left(\mathcal{E}^{p}\right)$ are equal to one. Let $C \in \operatorname{Fix}_{G}\left(\mathcal{E}^{p}\right)$ be an eigenvector of $\mathcal{A}_{p}$ with eigenvalue $\lambda \in[-1,1]$. We may assume that $C=\langle f\rangle \in \partial \mathcal{L}^{p}$, where $f: S^{m} \rightarrow S_{V}$ is a full $p$-eigenmap of boundary type. Since $\langle f\rangle \in \operatorname{Fix}_{G}\left(\mathcal{E}^{p}\right)$, $f$ is equivariant with respect to a homomorphism $\rho: G \rightarrow S O(V)$. Let $X \in \mathcal{G}$. Substituting $g=\exp (t X), t \in \mathbf{R}$, into (7) and differentiating (at $t=0$ ), we obtain

$$
X^{*}(f)=-R(X) \circ f,
$$

where $R: \mathcal{G} \rightarrow s o(V)$ is the differential of $\rho$. Viewing $R$ as a linear map $R: V \rightarrow$ $V \otimes \mathcal{G}^{*}$, we have

$$
\hat{f}=-\lambda_{p}^{-1 / 2} R \circ f .
$$

It follows that $V_{\hat{f}} \subset V_{f}$ holds for the corresponding spaces of components. In particular, $\hat{f}$ is of boundary type. Since $\langle\hat{f}\rangle=\mathcal{A}_{p}(\langle f\rangle)=\lambda\langle f\rangle$ this is possible only if $\lambda=1$ or if $\lambda<0$. The latter, however, contradicts to the Connecting Lemma.

For the converse, we assume that $\operatorname{Fix}_{G}\left(\mathcal{E}^{p}\right)$ is properly contained in the +1 eigenspace of $\mathcal{A}_{p}$. Let $f: S^{m} \rightarrow S_{V}$ be a full $p$-eigenmap of boundary type such 
that $\langle\hat{f}\rangle=\mathcal{A}_{p}(\langle f\rangle)=\langle f\rangle$ and assume that $\langle f\rangle \perp \operatorname{Fix}_{G}\left(\mathcal{E}^{p}\right)$. Since $\langle\hat{f}\rangle=\langle f\rangle$, there exists a linear homothety $R: V \rightarrow V \otimes \mathcal{G}^{*}$ such that

$$
X^{*}(f)=-R(X) \circ f, \quad X \in \mathcal{G} .
$$

In particular, the space of components $V_{f}=V_{\hat{f}}$ is a $\mathcal{G}$-submodule of $\mathcal{H}^{p}$. Thus, $V_{f} \subset \mathcal{H}^{p}$ is also a $G$-submodule.

Let $\left\{f_{0}^{l}\right\}_{l=0}^{n} \subset V_{f}, n+1=\operatorname{dim} V_{f}$, be an orthonormal basis and define $f_{0}: S^{m} \rightarrow$ $V_{f}$ by

$$
f_{0}(x)=\sum_{l=0}^{n} f_{0}^{l}(x) f_{0}^{l} .
$$

By construction, $f_{0}$ is $G$-equivariant (with respect to the $G$-module structure on $V_{f}$ ) and, since $G$ acts transitively on $S^{m}$, up to scaling, $f_{0}$ maps $S^{m}$ to the unit sphere of $V_{f}$. After scaling, we obtain a full $p$-eigenmap $f_{0}: S^{m} \rightarrow S_{V_{f}}$. By definition, we have $V_{f_{0}}=V_{f}$. Since $f_{0}$ is $G$-equivariant, $\left\langle f_{0}\right\rangle$ is fixed by $G$.

Consider the ray emanating from $\langle f\rangle$ through $\left\langle f_{0}\right\rangle$. Since $\mathcal{L}^{p}$ is compact and convex, this ray intersects $\mathcal{L}^{p}$ in a segment with endpoint beyond $\left\langle f_{0}\right\rangle$ represented by a full $p$-eigenmap $f^{\prime}: S^{m} \rightarrow S_{V^{\prime}}$. Since $f$ and $f_{0}$ have the same range dimension, $\operatorname{dim} V^{\prime}<\operatorname{dim} V$; in particular, $f_{0}$ and $f^{\prime}$ are incongruent. (We are implicitly using here the stratification of $\mathcal{L}^{p}$ induced by the inclusion relation among the spaces of components of eigenmaps; for details, cf. [13].)

Let $C$ be the center of mass of the $G$-orbit through $\langle f\rangle$. Clearly, $C$ is $G$-fixed. Moreover, since $\langle f\rangle$ is orthogonal to the $G$-module $\operatorname{Fix}_{G}\left(\mathcal{E}^{p}\right)$, so is $C$. Thus, $C=0$.

Let $C^{\prime}$ be the center of mass of the $G$-orbit through $\left\langle f^{\prime}\right\rangle$. As before, $C^{\prime} \in$ $\operatorname{Fix}_{G}\left(\mathcal{E}^{p}\right)$. Moreover, by convexity, $C^{\prime} \in \mathcal{L}^{p}$. Since $\left\langle f_{0}\right\rangle$ is $G$-fixed and is between $\langle f\rangle$ and $\left\langle f^{\prime}\right\rangle$, the point $\left\langle f_{0}\right\rangle$ is between $C=0$ and $C^{\prime}$ in $\mathcal{L}^{p}$. Thus, $f_{0}$ cannot be of boundary type; a contradiction. The lemma follows.

The last statement of Theorem 1 follows from the following:

Lemma 5. The +1 eigenspace of $\left(\mathcal{A}_{p}\right)^{2}$ is contained in $\operatorname{Fix}_{[G, G]}\left(\mathcal{E}^{p}\right)$.

Proof. Assume that $\left(\mathcal{A}_{p}\right)^{2}$ fixes $C \in \partial \mathcal{L}^{p}$. Let $f: S^{m} \rightarrow S_{V}$ be a full $p$-eigenmap of boundary type that represents $C$. By construction, $V_{f}$ is invariant under the second order differential operators $X^{*} Y^{*}, X, Y \in \mathcal{G}$. In particular, $V_{f}$ is a $[\mathcal{G}, \mathcal{G}]$ submodule of $\mathcal{H}^{p}$. As before, $V_{f}$ is a $[G, G]$-submodule. Since $G$ acts transitively on $S^{m}$, so does $[G, G]$. The previous proof now applies.

\section{Eigenmaps and Spherical Minimal Immersions}

A $p$-eigenmap $f: S^{m} \rightarrow S_{V}$ is a spherical minimal immersion of degree $p$ iff it is homothetic; that is, it satisfies (1). Thus, to prove Theorem 2, we need to show that $f: S^{m} \rightarrow S_{V}$ homothetic implies that $\hat{f}: S^{m} \rightarrow S_{V \otimes \mathcal{G}^{*}}$ is also homothetic. We now recall some results from [14] that will greatly reduce the amount of computations. Let $f: S^{m} \rightarrow S_{V}$ be a full $p$-eigenmap. We define the symmetric 2 -tensor $\Psi(f)$ on $S^{m}$ by

$$
\begin{aligned}
\Psi(f)(X, Y) & =\left\langle f_{*}(X), f_{*}(Y)\right\rangle-\lambda_{p} / m\langle X, Y\rangle \\
& =\left\langle f_{*}(X), f_{*}(Y)\right\rangle-\left\langle\left(f_{p}\right)_{*}(X),\left(f_{p}\right)_{*}(Y)\right\rangle,
\end{aligned}
$$

where $X, Y$ are vector fields on $S^{m}$ (and vectors tangent to $S^{m}$ are identified with their translates to the origin of $\left.\mathbf{R}^{m+1}\right)$. By definition, $f$ is homothetic iff $\Psi(f)=0$. 
We restrict $\Psi(f)$ to conformal vector fields on $S^{m}$; given $a \in \mathbf{R}^{m+1}$, the conformal vector field $X^{a}$ on $S^{m}$ is defined by

$$
X_{x}^{a}=a-\langle a, x\rangle, \quad x \in S^{m} .
$$

Since conformal vector fields span each tangent space in $S^{m}$, we see that $f$ is conformal iff $\Psi(a, b):=\Psi(f)\left(X^{a}, X^{b}\right)=0$ for all $a, b \in \mathbf{R}^{m+1}$. We now extend all objects to $\mathbf{R}^{m+1}$; the spherical harmonics to harmonic $p$-homogeneous polynomials in $m+1$ variables, the $p$-eigenmaps to harmonic $p$-homogeneous polynomial maps $f: \mathbf{R}^{m+1} \rightarrow V$ (that map the unit sphere to the unit sphere), the conformal vector field $X^{a}, a \in \mathbf{R}^{m+1}$, to $\mathbf{R}^{m+1}$ by

$$
X_{x}^{a}=a-\frac{\langle a, x\rangle}{\rho^{2}} x, \quad \rho^{2}=|x|^{2}, x \in \mathbf{R}^{m+1},
$$

and $\Psi(f)$ to $\mathbf{R}^{m+1}$ by

$$
\Psi(f)(a, b)=\left\langle f_{*}\left(X^{a}\right), f_{*}\left(X^{b}\right)\right\rangle-\lambda_{p} / m\left\langle X^{a}, X^{b}\right\rangle \rho^{2(p-1)}, a, b \in \mathbf{R}^{m+1} .
$$

Computations show [14] that $\Psi(f)(a, b)$ is a homogeneous polynomial of degree $2(p-1)$; in fact, we have

$$
\begin{aligned}
\Psi(f)(a, b) & =\left\langle\partial_{a} f, \partial_{b} f\right\rangle \\
& +\left(\lambda_{p} / m-p^{2}\right)\langle a, x\rangle\langle b, x\rangle \rho^{2(p-2)}-\lambda_{p} / m\langle a, b\rangle \rho^{2(p-1)},
\end{aligned}
$$

where $\partial_{a}$ denotes the directional derivative at the direction $a \in \mathbf{R}^{m+1}$.

Let $G \subset S O(m+1)$ be a closed subgroup acting transitively on $S^{m}$ and let $\mathcal{G}$ denote its Lie algebra. The elements of $\mathcal{G}$ will be considered as skew-symmetric matrices acting on $\mathbf{R}^{m+1}$. By (8), to prove Theorem 2, we need to show that, for $f: S^{m} \rightarrow S_{V}$ homothetic, $\left|\partial_{a} \hat{f}\right|^{2}, a \in \mathbf{R}^{m+1}$, is independent of $f$, so that it is then equal to $\left|\partial_{a} \hat{f}_{p}\right|^{2}$ and thereby cancel in $\Psi(\hat{f})(a, a)$.

Lemma 6. For $a \in \mathbf{R}^{m+1}$ and $X \in \mathcal{G}$, we have

$$
\left[\partial_{a}, X^{*}\right]=\partial_{X a}
$$

Proof. Let $x_{k}: \mathbf{R}^{m+1} \rightarrow \mathbf{R}, k=0, \ldots, m$, denote the $k$-th projection. We have $\partial_{a} x_{k}=a_{k}$ and, for $x \in \mathbf{R}^{m+1}$,

$$
X_{x}^{*}=\partial_{X x}=\sum_{k=0}^{m} x_{k} \partial_{X e_{k}}
$$

where $\left\{e_{k}\right\}_{k=0}^{m} \subset \mathbf{R}^{m+1}$ is the standard basis. With these, we compute

$$
\begin{aligned}
{\left[\partial_{a}, X^{*}\right] } & =\partial_{a} X^{*}-X^{*} \partial_{a} \\
& =\sum_{k=0}^{m} \partial_{a}\left(x_{k} \partial_{X e_{k}}\right)-\sum_{k=0}^{m} x_{k} \partial_{X e_{k}} \partial_{a} \\
& =\sum_{k=0}^{m}\left(\partial_{a} x_{k}\right) \partial_{X e_{k}}=\partial_{X a} .
\end{aligned}
$$

Lemma 7. For $a \in \mathbf{R}^{m+1}$ and $X \in \mathcal{G}$, we have

$$
X^{*} \partial_{X a}=1 / 2\left(-\partial_{X^{2} a}+\partial_{a}\left(X^{*}\right)^{2}-\left(X^{*}\right)^{2} \partial_{a}\right) .
$$


Proof. By Lemma 6, $\left[X^{*}, \partial_{X a}\right]=-\partial_{X^{2} a}$. Using this and Lemma 6 repeatedly, we compute

$$
\begin{aligned}
X^{*} \partial_{X a}+\partial_{X^{2} a} & =\partial_{X a} X^{*} \\
& =\left[\partial_{a}, X^{*}\right] X^{*} \\
& =\partial_{a}\left(X^{*}\right)^{2}-X^{*} \partial_{a} X^{*} \\
& =\partial_{a}\left(X^{*}\right)^{2}-X^{*}\left(\left[\partial_{a}, X^{*}\right]+X^{*} \partial_{a}\right) \\
& =\partial_{a}\left(X^{*}\right)^{2}-X^{*} \partial_{X a}-\left(X^{*}\right)^{2} \partial_{a} .
\end{aligned}
$$

Lemma 7 follows.

We now turn to the main computation. Let $\left\{E_{i}\right\}_{i=1}^{s} \subset \mathcal{G}$ be an orthonormal basis as before. We have

$$
\begin{aligned}
\lambda_{p}\left|\partial_{a} \hat{f}\right|^{2} & =\sum_{i=1}^{s}\left|\partial_{a} E_{i}^{*}(f)\right|^{2} \\
& =\sum_{i=1}^{s}\left|\left[\partial_{a}, E_{i}^{*}\right] f+E_{i}^{*} \partial_{a} f\right|^{2} \\
& =\sum_{i=1}^{s}\left|\partial_{E_{i} a} f+E_{i}^{*} \partial_{a} f\right|^{2} \\
& =\sum_{i=1}^{s}\left|\partial_{E_{i} a} f\right|^{2}+2 \sum_{i=1}^{s}\left\langle\partial_{E_{i} a} f, E_{i}^{*} \partial_{a} f\right\rangle+\sum_{i=1}^{s}\left|E_{i}^{*} \partial_{a} f\right|^{2} .
\end{aligned}
$$

We write the result as $I+2 I I+I I I$. Assume now that $f$ is homothetic so that $\Psi(f)=0$. In particular, $\sum_{i=1}^{s} \Psi(f)\left(E_{i} a, E_{i} a\right)=0$ so that, by (8), $I$ is independent of $f$. Using skew symmetry of $X^{*}, X \in \mathcal{G}$, on $\mathcal{H}^{p-1}$ and Lemma 7 , we have

$$
\begin{aligned}
I I & =-\sum_{i=1}^{s}\left\langle E_{i}^{*} \partial_{E_{i} a} f, \partial_{a} f\right\rangle \\
& =1 / 2 \sum_{i=1}^{s}\left\langle\partial_{E_{i}^{2} a} f, \partial_{a} f\right\rangle \\
& -1 / 2\left\langle\partial_{a} \sum_{i=1}^{s}\left(E_{i}^{*}\right)^{2} f, \partial_{a} f\right\rangle+1 / 2\left\langle\sum_{i=1}^{s}\left(E_{i}^{*}\right)^{2} \partial_{a} f, \partial_{a} f\right\rangle \\
& =1 / 2\left\langle\partial_{\beta(a)} f, \partial_{a} f\right\rangle+\left(\lambda_{p}-\lambda_{p-1}\right) / 2\left\langle\partial_{a} f, \partial_{a} f\right\rangle,
\end{aligned}
$$

where $\beta(a)=\sum_{i=1}^{s} E_{i}^{2} a, a \in \mathbf{R}^{m+1}$. Every term here is independent of $f$ since $\Psi(f)=0$. Finally, we have

$$
I I I=-\left\langle\sum_{i=1}^{s}\left(E_{i}^{*}\right)^{2} \partial_{a} f, \partial_{a} f\right\rangle=\lambda_{p-1}\left\langle\partial_{a} f, \partial_{a} f\right\rangle
$$

and this is also independent of $f$. Theorem 2 is proved.

\section{Raising the Degree}

As noted in Section 1, it is enough to prove Theorem 3 for $q=p+1$. We first define an $S O(m+1)$-equivariant imbedding of $\mathcal{L}^{p}$ into $\mathcal{L}^{p+1}$, that extends to a linear inclusion $\mathcal{E}^{p} \subset \mathcal{E}^{p+1}$ via (2). The imbedding is given on the level of eigenmaps by 
the degree raising operator [14] as follows: Given a full p-eigenmap $f: S^{m} \rightarrow S_{V}$, we define $f^{+}: S^{m} \rightarrow S_{V \otimes \mathcal{H}^{1}}$ by

$$
f^{+}=\sqrt{\lambda_{2 p} /\left(2 \lambda_{p}\right)} \sum_{k=0}^{m} H\left(x_{k} f\right) \otimes y_{k}
$$

where $H$ is the harmonic projection operator [16] and $f$ is considered as a harmonic polynomial map $f: \mathbf{R}^{m+1} \rightarrow V$. The correspondence $f \mapsto f^{+}$gives rise to the linear imbedding of the moduli spaces in question; in fact, it extends to a linear $S O(m+1)$ module monomorphism $\Phi_{p}^{+}: S^{2}\left(\mathcal{H}^{p}\right) \rightarrow S^{2}\left(\mathcal{H}^{p+1}\right)$. By definition, $\Phi_{p}^{+}(\langle f\rangle)=\left\langle f^{+}\right\rangle$. To make $\Phi_{p}^{+}$more explicit, we first note that $\mathcal{H}^{p+1}$ is contained in $\mathcal{H}^{p} \otimes \mathcal{H}^{1}$ as an $S O(m+1)$-submodule via the inclusion $\iota_{p}: \mathcal{H}^{p+1} \rightarrow \mathcal{H}^{p} \otimes \mathcal{H}^{1}$ given by

$$
\iota_{p}(h)=c_{p} \sum_{k=0}^{m} \partial_{k} h \otimes y_{k}, \quad h \in \mathcal{H}^{p+1},
$$

where $\partial_{k}$ denotes the $k$-th partial derivative. The value of the constant $c_{p}$ is determined by the condition

$$
\iota_{p}^{\top} \circ \iota_{p}=I
$$

We have

$$
\Phi_{p}^{+}(C)=\iota_{p}^{\top} \circ(C \otimes I) \circ \iota_{p}, \quad C \in S^{2}\left(\mathcal{H}^{p}\right) .
$$

For further details, cf. [14]. The partial differentiation $\partial_{k}$, as a linear map $\mathcal{H}^{p+1} \rightarrow$ $\mathcal{H}^{p}$ has transpose $H\left(x_{k} \cdot\right): \mathcal{H}^{p} \rightarrow \mathcal{H}^{p+1}$ multiplied by $(p+1) \lambda_{2 p} /\left(2 \lambda_{p}\right)$ [14]. Simple computation shows that

$$
\iota_{p}^{\top}\left(h^{\prime} \otimes y_{k}\right)=\sqrt{\lambda_{2 p} /\left(2 \lambda_{p}\right)} H\left(x_{k} h^{\prime}\right), \quad h^{\prime} \in \mathcal{H}^{p}, k=0, \ldots, m .
$$

The normalizing condition (9) now gives

$$
c_{p}=\frac{1}{\sqrt{p+1}} \sqrt{\frac{2 \lambda_{p}}{\lambda_{2 p}}} .
$$

Theorem 3 will follow if we prove the following:

Lemma 8. Let $G \subset S O(m+1)$ be a closed subgroup acting transitively on $S^{m}$. Then, for $C \in S^{2}\left(\mathcal{H}^{p}\right)$, we have

$$
\lambda_{p+1}\left(\mathcal{A}_{p+1}\left(\Phi_{p}^{+}(C)\right)-\Phi_{p}^{+}(C)\right)=\lambda_{p}\left(\Phi_{p}^{+}\left(\mathcal{A}_{p}(C)\right)-\Phi_{p}^{+}(C)\right) .
$$

Proof. Given $C \in S^{2}\left(\mathcal{H}^{p}\right)$, we work out

$$
\lambda_{p+1} \mathcal{A}_{p+1}\left(\Phi_{p}^{+}(C)\right)-\lambda_{p} \Phi_{p}^{+}\left(\mathcal{A}_{p}(C)\right) .
$$

For $h \in \mathcal{H}^{p+1}$, we have

$$
\begin{aligned}
\lambda_{p+1} \mathcal{A}_{p+1}\left(\Phi_{p}^{+}(C)\right)(h) & =\lambda_{p+1} \alpha_{p+1}^{\top}\left(\Phi_{p}^{+}(C) \otimes I\right) \alpha_{p+1}(h) \\
& =-\sum_{i=1}^{s} E_{i}^{*}\left(\Phi_{p}^{+}(C) E_{i}^{*} h\right) \\
& =-c_{p} \sum_{i=1}^{s} \sum_{k=0}^{m} E_{i}^{*}\left(\iota_{p}^{\top}(C \otimes I) \partial_{k}\left(E_{i}^{*} h\right) \otimes y_{k}\right) \\
& =-(p+1)^{-1 / 2} \sum_{i=1}^{s} \sum_{k=0}^{m} H\left(E_{i}^{*}\left(x_{k} C\left(\partial_{k}\left(E_{i}^{*} h\right)\right)\right),\right.
\end{aligned}
$$


where we used (10)-(11). On the other hand, we have

$$
\begin{aligned}
\lambda_{p} \Phi_{p}^{+}\left(\mathcal{A}_{p}(C)\right)(h) & =\lambda_{p} \iota_{p}^{\top}\left(\mathcal{A}_{p}(C) \otimes I\right) \iota_{p}(h) \\
& =\lambda_{p} c_{p} \sum_{k=0}^{m} \iota_{p}^{\top}\left(\mathcal{A}_{p}(C) \otimes I\right)\left(\partial_{k} h \otimes y_{k}\right) \\
& =\lambda_{p}(p+1)^{-1 / 2} \sum_{k=0}^{m} H\left(x_{k} \mathcal{A}_{p}(C)\left(\partial_{k} h\right)\right) \\
& =-(p+1)^{-1 / 2} \sum_{i=1}^{s} \sum_{k=0}^{m} H\left(x_{k} E_{i}^{*} C\left(E_{i}^{*}\left(\partial_{k} h\right)\right)\right) .
\end{aligned}
$$

The difference (12) of these two terms is

$$
-(p+1)^{-1 / 2} \sum_{i=1}^{s} \sum_{k=0}^{m}\left(H\left(E_{i}^{*}\left(x_{k} C\left(\partial_{k} E_{i}^{*} h\right)\right)\right)-H\left(x_{k} E_{i}^{*} C\left(E_{i}^{*} \partial_{k} h\right)\right)\right) .
$$

Performing differentiation in the first term, the double sum (without the coefficient) rewrites as

$$
\sum_{i=1}^{s} \sum_{k=0}^{m} H\left(E_{i}^{*}\left(x_{k}\right) C\left(\partial_{k} E_{i}^{*} h\right)\right)+\sum_{i=1}^{s} \sum_{k=0}^{m} H\left(x_{k} E_{i}^{*} C\left[\partial_{k}, E_{i}^{*}\right] h\right) .
$$

We write this as $I+I I$ and work out each term separately. Using the standard basis $\left\{e_{k}\right\}_{k=0}^{m} \subset \mathbf{R}^{m+1}$, we have

$$
\begin{aligned}
I & =\sum_{i=1}^{s} \sum_{k=0}^{m} H\left(\left\langle E_{i} x, e_{k}\right\rangle C\left(\partial_{k} E_{i}^{*} h\right)\right) \\
& =\sum_{i=1}^{s} \sum_{k, l=0}^{m} H\left(x_{l}\left\langle E_{i} e_{l}, e_{k}\right\rangle C\left(\partial_{k} E_{i}^{*} h\right)\right) \\
& =\sum_{i=1}^{s} \sum_{l=0}^{m} H\left(x_{l} C\left(\partial_{E_{i} e_{l}} E_{i}^{*} h\right)\right) .
\end{aligned}
$$

By Lemmas 6-7, the second-order operator

$$
\begin{aligned}
\partial_{E_{i} e_{l}} E_{i}^{*} & =\left[\partial_{E_{i} e_{l}}, E_{i}^{*}\right]+E_{i}^{*} \partial_{E_{i} e_{l}} \\
& =1 / 2\left(\partial_{E_{i}^{2} e_{l}}+\partial_{l}\left(E_{i}^{*}\right)^{2}-\left(E_{i}^{*}\right)^{2} \partial_{l}\right)
\end{aligned}
$$

Summing up with respect to $i=1, \ldots, s$, and using that $\sum_{i=1}^{s}\left(E_{i}^{*}\right)^{2}=-\lambda_{p} I$ on $\mathcal{H}^{p}$, we arrive at

$$
I=1 / 2 \sum_{l=0}^{m} H\left(x_{l} C \partial_{\beta\left(e_{l}\right)} h\right)-\left(\lambda_{p+1}-\lambda_{p}\right) / 2 \sum_{l=0}^{m} H\left(x_{l} C \partial_{l} h\right),
$$

where $\beta(a)=\sum_{i=1}^{s} E_{i}^{2} a, a \in \mathbf{R}^{m+1}$. Using Lemma 6 , the second sum in (13) rewrites as

$$
I I=\sum_{i=1}^{s} \sum_{k=0}^{m} H\left(x_{k} E_{i}^{*} C\left[\partial_{k}, E_{i}^{*}\right] h\right)=\sum_{i=1}^{s} \sum_{k=0}^{m}\left\langle E_{i} e_{k}, e_{l}\right\rangle H\left(x_{k} E_{i}^{*} C \partial_{l} h\right) .
$$

For $l=0, \ldots, m$ fixed, consider the operator

$$
\mathcal{K}_{l}: h^{\prime} \mapsto \sum_{i=1}^{s} \sum_{k=0}^{m}\left\langle E_{i} e_{k}, e_{l}\right\rangle H\left(x_{k} E_{i}^{*} h^{\prime}\right), \quad h^{\prime} \in \mathcal{H}^{p} .
$$


The simplest way to work this out is to simplify its transpose. As noted above, up to a constant multiple, the transpose of $H\left(x_{k} \cdot\right)$ is $\partial_{k}$, so that, by Lemma 7, for $h^{\prime \prime} \in \mathcal{H}^{p}$, we have

$$
\begin{aligned}
\mathcal{K}_{l}^{\top}\left(h^{\prime \prime}\right) & =2(p+1) \lambda_{p} / \lambda_{2 p} \sum_{i=1}^{s} \sum_{k=0}^{m}\left\langle e_{k}, E_{i} e_{l}\right\rangle E_{i}^{*} \partial_{k} h^{\prime \prime} \\
& =2(p+1) \lambda_{p} / \lambda_{2 p} \sum_{i=1}^{s} E_{i}^{*} \partial_{E_{i} e_{l}} h^{\prime \prime} \\
& =2(p+1) \lambda_{p} / \lambda_{2 p}\left(-\partial_{\beta\left(e_{l}\right)} h^{\prime \prime}+\left(\lambda_{p}-\lambda_{p+1}\right) \partial_{l} h^{\prime \prime}\right) .
\end{aligned}
$$

Taking transpose again, we obtain

$$
\mathcal{K}_{l}\left(h^{\prime}\right)=-(1 / 2) H\left(\left\langle\beta\left(e_{l}\right), x\right\rangle h^{\prime}\right)-(1 / 2)\left(\lambda_{p+1}-\lambda_{p}\right) H\left(x_{l} h^{\prime}\right), \quad h^{\prime} \in \mathcal{H}^{p} .
$$

Using this, we have

$$
I I=-(1 / 2) \sum_{l=0}^{m} H\left(\left\langle\beta\left(e_{l}\right), x\right\rangle C \partial_{l} h\right)-(1 / 2)\left(\lambda_{p+1}-\lambda_{p}\right) \sum_{i=1}^{s} \sum_{l=0}^{m} H\left(x_{l} C \partial_{l} h\right) .
$$

Combining this with (14), we arrive at

$$
I+I I=-\left(\lambda_{p+1}-\lambda_{p}\right) \sum_{l=0}^{m} H\left(x_{l} C \partial_{l} h\right) .
$$

since the first terms in $I$ and $I I$ cancel by symmetry of $\beta$. We finally obtain that the difference in (12) is equal to

$$
\left(\lambda_{p+1}-\lambda_{p}\right) / \sqrt{p+1} \sum_{l=0}^{m} H\left(x_{l} C \partial_{l} h\right)=\left(\lambda_{p+1}-\lambda_{p}\right) \Phi_{p}^{+}(C)(h)
$$

and Theorem 3 follows.

\section{Estimates on the Range Dimension}

Let $G$ be semisimple and $f: S^{m} \rightarrow S_{V}$ a full $p$-eigenmap of boundary type. Assume that $C=\langle f\rangle$ is contained in an $\mathcal{A}_{p}$-invariant $G$-submodule $\mathcal{V}$ of $\mathcal{E}^{p}$. Let $\lambda_{1}, \ldots, \lambda_{d}$ denote the distinct eigenvalues of $\mathcal{A}_{p} \mid \mathcal{V}$. Since $\mathcal{V}$ is orthogonal to $\operatorname{Fix}_{G}\left(\mathcal{E}^{p}\right)$, we have $\lambda_{j} \in(-1,1), j=1, \ldots, d$. Let $C^{k}=\left(\mathcal{A}_{p}\right)^{k}(C), k=0, \ldots, d$; $C^{0}=C$. We may assume that $C^{d} \in \partial \mathcal{L}^{p}$ since otherwise $e(f) \leq d$ and the lower estimate (6) applies.

We claim that the entire simplex with vertices $C^{1}, \ldots, C^{d}$ is contained in the boundary of $\mathcal{L}^{p}$. This can be seen as follows. The space $V_{\hat{f}}$ of components of $\hat{f}$ is spanned by the components of $X^{*}(f), X \in \mathcal{G}$. Similarly, $V_{\hat{f}^{2}}$ is spanned by the components of $X^{*} Y^{*}(f), X, Y \in \mathcal{G}$. Since $G$ is semisimple, it follows that $V_{\hat{f}} \subset V_{\hat{f}^{2}}$. By the Connecting Lemma, the segment joining $C^{1}$ and $C^{2}$ is on the boundary of $\mathcal{L}^{p}$ provided that $C^{2} \in \partial \mathcal{L}^{p}$. Iterating ${ }^{\wedge}$, we have

$$
V_{\hat{f}} \subset V_{\hat{f}^{2}} \subset \ldots \subset V_{\hat{f}^{d}}
$$

and, since $\hat{f}^{k}$ corresponds to $C^{k}, k=1, \ldots, d$, by the Connecting Lemma, $V_{\hat{f}^{d}}$ contains the spaces of components of all eigenmaps that correspond to points in the simplex with vertices $C^{1}, \ldots, C^{d}$. 
Next we claim that the simplex with vertices $C=C^{0}$ and $C^{1}, \ldots, C^{d}$ intersects the interior of $\mathcal{L}^{p}$. To prove this, we first notice that $C^{0}, \ldots, C^{d}$ are linearly dependent. More specifically, we have

$$
\sum_{k=0}^{d}(-1)^{k} s_{k}\left(\lambda_{1}, \ldots, \lambda_{d}\right) C^{d-k}=0,
$$

where $s_{k}$ is the $k$-th elementary symmetric polynomial in $d$ variables. To show this, we write $C=\sum_{j=1}^{d} X_{j}$, where $X_{j}$ is an eigenvector of $\mathcal{A}_{p}$ with eigenvalue $\lambda_{j}$. Applying $\left(\mathcal{A}_{p}\right)^{d-k}$ to both sides, we have $C^{d-k}=\sum_{j=1}^{d} \lambda_{j}^{d-k} X_{j}$. Now (15) follows from the identity

$$
\sum_{k=0}^{d}(-1)^{k} s_{k}\left(\lambda_{1}, \ldots, \lambda_{d}\right) \lambda_{j}^{d-k}=\left.\left(x-\lambda_{1}\right) \ldots\left(x-\lambda_{d}\right)\right|_{x=\lambda_{j}}=0 .
$$

We now rearrange (15) into convex linear combinations. For simplicity, we set $a_{k}=(-1)^{k} s_{k}\left(\lambda_{1}, \ldots, \lambda_{d}\right)$. Let

$$
I^{+}=\left\{k \mid a_{k}>0\right\}, I^{-}=\left\{k \mid a_{k}<0\right\}
$$

and

$$
S^{+}=\sum_{k \in I^{+}} a_{k}, S^{-}=\sum_{k \in I^{-}}\left(-a_{k}\right) .
$$

By Theorem 1, we have

$$
S^{+}-S^{-}=\sum_{k=0}^{d} a_{k}=\left(1-\lambda_{1}\right) \ldots\left(1-\lambda_{d}\right)>0 .
$$

Clearly, $0 \in I^{+}$. We may assume that $I^{-}$is nonempty. Indeed, if $I^{-}=\emptyset$ then, by (15), we have

$$
\sum_{k \in I^{+}} \frac{a_{k}}{S^{+}} C^{d-k}=0
$$

This equality shows that a convex linear combination of the vertices $C^{d-k}, k \in I^{+}$, gives the origin; an interior point of $\mathcal{L}^{p}$.

Assuming $I^{ \pm} \neq \emptyset$, we have $S^{ \pm}>0$. Rewriting (15), we have

$$
\frac{S^{+}}{S^{-}} \sum_{k \in I^{+}} \frac{a_{k}}{S^{+}} C^{d-k}=\sum_{k \in I^{-}} \frac{\left(-a_{k}\right)}{S^{-}} C^{d-k} .
$$

The right-hand-side is a point in the simplex with vertices $C^{d-k}, k \in I^{-}$. By the inequality above, $S^{+} / S^{-}>1$, so that the sum on the left-hand-side must be in the interior of $\mathcal{L}^{p}$. But this sum is a point in the simplex with vertices $C^{d-k}, k \in I^{+}$. The claim follows.

Let $d=1$. Since the segment joining $C^{0}=\langle f\rangle$ and $C^{1}=\langle\hat{f}\rangle$ contains a point in the interior of $\mathcal{L}^{p}$, by the Connecting Lemma, we have $V_{f}+V_{\hat{f}}=\mathcal{H}^{p}$. Since

$$
\begin{aligned}
\operatorname{dim} \mathcal{H}^{p} & \leq \operatorname{dim} V_{f}+\operatorname{dim} V_{\hat{f}} \\
& \leq \operatorname{dim} V+\operatorname{dim} V \cdot \operatorname{dim} \mathcal{G}^{*} \\
& =\operatorname{dim} V(1+\operatorname{dim} G)
\end{aligned}
$$

the first statement of Theorem 4 follows. 
Let $d \geq 2$. We first notice that, with respect to an orthonormal basis $\left\{E_{i}\right\}_{i=1}^{s} \subset$ $\mathcal{G}$, we have

$$
f=1 / \lambda_{p} \triangle^{S^{m}} f=-1 / \lambda_{p} \sum_{i=1}^{s}\left(E_{i}^{*}\right)^{2} f .
$$

Taking components, we obtain $V_{f} \subset V_{\hat{f}^{2}}$. By the Connecting Lemma, the entire simplex with vertices $C^{0}, \ldots, C^{d}$ is contained in the boundary of $\mathcal{L}^{p}$. This contradicts to the previous claim and shows that $C^{d}$ must be in the interior of $\mathcal{L}^{p}$. Thus $e(f) \leq d$ and Theorem 4 follows.

\section{EXAMPLES}

Let $m=3$. We have the standard splitting $S O(4)=S U(2) \cdot S U(2)^{\prime}$, where $S U(2) \cap S U(2)^{\prime}=\{ \pm I\}$ and $S U(2)^{\prime}$ is obtained from $S U(2)$ by conjugating with the diagonal matrix in $S O(4)$ with diagonal entries $1,1,1,-1$. We will use the following notation: If $W$ is an $S U(2)$-module, then $W^{\prime}$ denotes the $S U(2)^{\prime}$-module obtained from $W$ by conjugating $S U(2)^{\prime}$ to $S U(2)$ within $S O(4)$ with the diagonal matrix above, and then applying the $S U(2)$-module structure to $W$. If, in addition, $-I$ acts on $W^{\prime}$ trivially, then $W^{\prime}$ is also considered as an $S O(4)$-module with trivial action of $S U(2)$ on $W^{\prime}$. We use similar notation when the roles of $S U(2)$ and $S U(2)^{\prime}$ are switched.

Let $W_{p}$ denote the complex irreducible $S U(2)$-module of dimension $p+1$. For $p$ even, $W_{p}$ is the complexification of a real irreducible $S U(2)$-module $R_{p}$. For $p$ odd, $W_{p}$, considered as a real $S U(2)$-module, is irreducible. For $p$ even, we have

$$
\mathcal{H}^{p} \cong R_{p} \otimes R_{p}^{\prime}
$$

as real $S O(4)$-modules.

In [15] Ziller and the author proved that, for $u \geq v \geq 1$ and $u+v$ even, $V_{4}^{(u, v)}$ (cf. (2)) contains the trivial $S U(2)$-module iff $u=v$. In this case, $V_{4}^{(u, u)}$ is the complexification of the $S O(4)$-module $R_{2 u} \oplus R_{2 u}^{\prime}$. Combining this with (2), we see that

$$
\operatorname{Fix}_{S U(2)}\left(\mathcal{E}^{p}\right) \cong \sum_{k=1}^{[|p / 2|]} R_{4 k}^{\prime},
$$

as real $S U(2)^{\prime}$-modules. As noted above, $\mathcal{L}^{p}$ intersected with this linear subspace parametrizes the congruence classes of full $S U(2)$-equivariant $p$-eigenmaps.

Let $p=2$ and $G=S U(2)^{\prime}$. As shown in [12], the moduli space $\mathcal{L}^{2}$ is the convex hull of the slices $\operatorname{Fix}_{S U(2)}\left(\mathcal{L}^{2}\right)$ and $\operatorname{Fix}_{S U(2)^{\prime}}\left(\mathcal{L}^{2}\right)$. By Theorem $1, \mathcal{A}_{2}$ acts on the irreducible $S U(2)^{\prime}$-module $\operatorname{Fix}_{S U(2)}\left(\mathcal{E}^{2}\right)$ as multiplication by a real number $\lambda \in(-1,1)$, and as identity on $\operatorname{Fix}_{S U(2)^{\prime}}\left(\mathcal{E}^{2}\right)$. We claim that $\lambda=-1 / 2$. To show this, consider the Hopf map, Hopf : $S^{3} \rightarrow S^{2}$, given, in complex variables $z=x_{1}+i x_{2}$ and $w=x_{3}+i x_{4}, x=(z, w)=\left(x_{1}, x_{2}, x_{3}, x_{4}\right) \in \mathbf{C}^{2}=\mathbf{R}^{4}$, by

$$
\operatorname{Hopf}(z, w)=\left(|z|^{2}-|w|^{2}, 2 z \bar{w}\right), \quad|z|^{2}+|w|^{2}=1 .
$$

(As shown in [12], $\operatorname{Fix}_{S U(2)}\left(\mathcal{L}^{2}\right)$ is the convex hull of the orbit $S U(2)^{\prime}(\langle\operatorname{Hopf}\rangle)$, that is a minimally imbedded projective plane in its respective 4 -sphere.) The standard basis of the Lie algebra $s o(4)$ is given by the vectors $A_{i k}=x_{i} \partial_{k}-x_{k} \partial_{i}$, $0 \leq i<k \leq 3$. The Lie subalgebra $s u(2) \subset s o(4)$ is spanned by

$$
X=\left[\begin{array}{rr}
0 & 1 \\
-1 & 0
\end{array}\right], Y=\left[\begin{array}{cc}
0 & i \\
i & 0
\end{array}\right], Z=\left[\begin{array}{rr}
i & 0 \\
0 & -i
\end{array}\right] \text {. }
$$


We have

$$
X^{*}=A_{13}-A_{24}, \quad Y^{*}=A_{14}+A_{23}, \quad Z^{*}=A_{12}+A_{34} .
$$

Using complex coordinates and $\partial_{z}=1 / 2\left(\partial_{1}-i \partial_{2}\right), \partial_{\bar{z}}=1 / 2\left(\partial_{1}+i \partial_{2}\right)$ etc., we have

$$
\begin{aligned}
X^{*} & =-\bar{w} \partial_{z}-w \partial_{\bar{z}}+\bar{z} \partial_{w}+z \partial_{\bar{w}}, \\
Y^{*} & =i\left(-\bar{w} \partial_{z}+w \partial_{\bar{z}}+\bar{z} \partial_{w}-z \partial_{\bar{w}}\right), \\
Z^{*} & =i\left(z \partial_{z}-\bar{z} \partial_{\bar{z}}+w \partial_{w}-\bar{w} \partial_{\bar{w}}\right) .
\end{aligned}
$$

Now, easy computation shows that $\widehat{\text { Hopf }}$ is congruent to the complex Veronese map $\operatorname{Ver}^{\mathbf{C}}: S^{3} \rightarrow S^{5}$ given by $\operatorname{Ver}^{\mathbf{C}}(z, w)=\left(z^{2}, \sqrt{2} z w, w^{2}\right),(z, w) \in \mathbf{C}^{2}$. Since $\langle$ Hopf $\rangle$ and $\left\langle\operatorname{Ver}^{\mathbf{C}}\right\rangle$ are both contained in $\operatorname{Fix}_{S U(2)}\left(\mathcal{L}^{2}\right)$, we see that they are opposite points on the boundary of $\mathcal{L}^{2}$ and $\lambda$ is negative. Both quadratic eigenmaps have the property that their components are orthogonal with the same norm. Thus, the diagonal $(9 \times 9)$-matrix $\langle$ Hopf $\rangle$ consists of a diagonal $(3 \times 3)$-block with entries 2 and another diagonal $(6 \times 6)$-block with entries -1 . Similarly, $\left\langle\operatorname{Ver}^{\mathbf{C}}\right\rangle$ consists of a diagonal $(3 \times 3)$-block with entries -1 and a diagonal $(6 \times 6)$-block with entries $1 / 2$. We obtain that $|\langle\operatorname{Hopf}\rangle|=3 \sqrt{2}$ and $\left|\left\langle\operatorname{Ver}^{\mathrm{C}}\right\rangle\right|=3 / \sqrt{2}$. The ratio gives $\lambda=-1 / 2$. The iteration formula (5) immediately gives that, for $p \geq 2$, the eigenvalue of $\mathcal{A}_{p}$ on $R_{4}^{\prime} \subset \mathcal{E}^{p}$ is $1-12 /(p(p+2))$.

Let $p=4$ and $G=S U(2)^{\prime}$. The moduli space $\mathcal{M}^{4}$ is the convex hull of the slices $\operatorname{Fix}_{S U(2)}\left(\mathcal{F}^{4}\right)$ and $\operatorname{Fix}_{S U(2)^{\prime}}\left(\mathcal{F}^{4}\right)[15]$. By Theorem 1, $\mathcal{A}_{4}$ acts on the irreducible $S U(2)^{\prime}$-module $\operatorname{Fix}_{S U(2)}\left(\mathcal{F}^{4}\right)$ as multiplication by a real number $\lambda \in(-1,1)$, and as identity on $\operatorname{Fix}_{S U(2)^{\prime}}\left(\mathcal{F}^{4}\right)$. We claim that $\lambda=-2 / 3$. The verification of this is completely analogous to the case of quadratic eigenmaps. The role of the Hopf map is played by the $S U(2)$-equivariant minimal immersion $\mathcal{I}: S^{3} \rightarrow S^{9}[2,15]$ given by

$$
\begin{aligned}
\mathcal{I}(z, w)= & \left(1 / \sqrt{2}\left(z^{4}-\bar{w}^{4}\right), \sqrt{6} z^{2} \bar{w}^{2}\right. \\
& \sqrt{2}\left(z^{3} w-\bar{z} \bar{w}^{3}\right), \sqrt{6}\left(z \bar{z}^{2} w-\bar{z} w^{2} \bar{w}\right), \\
& \left.\sqrt{3 / 2}\left(z^{2} w^{2}-\bar{z}^{2} \bar{w}^{2}\right), 1 / \sqrt{2}\left(|z|^{4}-4|z|^{2}|w|^{2}+|w|^{4}\right)\right) .
\end{aligned}
$$

The components of $\mathcal{I}$ are orthogonal with the same norm. (The $S U(2)^{\prime}$-orbit of $\langle\mathcal{I}\rangle$ is a minimally imbedded octahedral manifold in the respective 8-sphere [15].)

Computation shows that $\hat{\mathcal{I}}$, made full, is a spherical minimal immersion with range $S^{14}$, and $\langle\mathcal{I}\rangle$ and $\langle\hat{\mathcal{I}}\rangle$ are opposite points on the boundary of $\mathcal{M}^{4}$ and $\lambda$ is negative. Computing lengths as before, we obtain $\lambda=-2 / 3$. By the iteration formula (5), $\mathcal{A}_{p}$ on $R_{8}^{\prime} \subset \mathcal{F}^{p}, p \geq 4$, has eigenvalue $1-40 /(p(p+2))$.

\section{REFERENCES}

1. Calabi E., Minimal immersions of surfaces in euclidean spheres, J. Diff. Geom., 1 (1967) 111-125. MR 38:1616

2. DeTurck D.-Ziller W., Minimal isometric immersions of spherical space forms in spheres, Comment. Math. Helvetici 67 (1992) 428-458. MR 93f:53050

3. DoCarmo M.-Wallach N., Minimal immersions of spheres into spheres, Ann. of Math., 93 (1971) 43-62. MR 43:4048

4. Eells J.-Sampson J.H., Harmonic mappings of Riemannian manifolds, Amer. J. Math. 86 (1964) 109-160. MR 29:1603

5. Ejiri, N., Totally real submanifolds in a 6-sphere, Proc. Amer. Math. Soc., 83 (1981) 759-763. MR 83a:53033 
6. Escher Ch., Minimal isometric immersions of inhomogeneous spherical space forms into spheres - A necessary condition for existence, Trans. Amer. Math.Soc., Vol.348, No.9, (1996) 3713-3732. MR 96m:53068

7. Escher Ch., On inhomogeneous space forms, Preprint, Oregon State University, 1997.

8. Gauchman H.-Toth, G., Fine structure of the space of spherical minimal immersions, Trans. Amer. Math. Soc. Vol.348, No.6 (1996) 2441-2463. MR 96i:53058

9. Mashimo K., Minimal immersions of 3-dimensional sphere into spheres, Osaka J. Math., 21 (1984) 721-732. MR 86d:53040

10. Moore J.D., Isometric immersions of space forms into space forms, Pacific J. Math., 40 (1976) 157-166. MR 46:4442

11. Takahashi T., Minimal immersions of Riemannian manifolds, J. Math. Soc. Japan, 18 (1966) 380-385. MR 33:6551

12. Toth G., Classification of quadratic harmonic maps of $S^{3}$ into spheres, Indiana U. Math. J., 36 (1987) 231-239. MR 89b:58058

13. Toth G., Harmonic maps and minimal immersions through representation theory, Academic Press, Boston, 1990. MR 91a:58050

14. Toth G., Eigenmaps and the space of minimal immersions between spheres, Indiana Univ. Math. J. 46 (1997) 637-658. MR 98k:53082

15. Toth G.-Ziller W., Spherical minimal immersions of the 3-sphere, Comment. Math. Helvetici (to appear).

16. Vilenkin N.I., Special Functions and the Theory of Group Representations, AMS Translations of Mathematical Monographs, Vol.22, 1968. MR 37:5429

17. Wallach N, Minimal immersions of symmetric spaces into spheres, in Symmetric Spaces, Dekker, New York (1972) 1-40. MR 53:11545

18. Wolf J., Spaces of Constant Curvature, McGraw-Hill, 1967. MR 36:829

Department of Mathematics, Rutgers University, Camden, New Jersey 08102

E-mail address: gtoth@crab.rutgers.edu 